\title{
Compromiso organizacional de los trabajadores de una Universidad Pública
}

\begin{abstract}
RESUMEN
Este artículo es un trabajo realizado en una muestra de 72 sujetos, con el objeto de conocer el compromiso de los trabajadores para con la organización donde laboran y la relación entre el compromiso organizacional y las variables demográficas. Los resultados muestran que la mayoría de ellos tienen un compromiso normal con su organización y que no existe relación con las variables demográficas analizadas. Un análisis cruzado entre los factores del compromiso organizacional arroja que existe relación significativa entre algunos de ellos.
\end{abstract}

Palabras Clave: Compromiso organizacional, trabajadores, universidad, variables demográficas.

ORganizational COMPROMISE OF PUBLIC UNIVERSITY EMPLEYEE'S ABSTRACT

This article a study carried out in a sample of 72 subjects in order to investigate the commitment of workers for organization where they work and the relationship between organizational commitment and demographic variables. The results show that the majority have a normal level of commitment to organization and that no relationships exist with the demographic variables used in this analysis. A cross-analysis between the factors of organizational commitment shows that a significant relationship exists between some of them.

Key Words: Organizational commitment, workers, university, demographic variables.

\section{INTRODUCCIÓN}

Una de las preocupaciones de los directivos de las organizaciones ha sido siempre, cómo lograr que sus trabajadores eleven su nivel de rendimiento para obtener una mayor productividad. Se han desarrollado un conjunto de investigaciones, a pesar de las dificultades y resistencias para invertir en estas tareas de parte de las organizaciones y Estados, especialmente en países Latinoamericanos. Sin embargo, una revisión detallada de la literatura dice que es difícil encontrar una investigación suficiente, necesaria y clara para afirmar que un trabajador satisfecho sea altamente productivo, por el contrario, en la práctica es posible encontrar trabajadores satisfechos y poco productivos.

Si para los investigadores de las organizaciones es un reto cómo elevar el rendimiento y la productividad, para los empresarios debe ser más que eso; lo que los directivos aún no comprenden es que contar con colaboradores con mística, espíritu de cuerpo o con la "camiseta bien puesta" (como dicen ellos), es mediante el esfuerzo conjunto entre los directivos y los investigadores y ello, hoy en día, más que una aspiración, es una meta que se debe lograr. Lo importante es que directivos y trabajadores entiendan que la única manera de alcanzar el progreso y el bienestar individual y de la organización es elevando el rendimiento y la productividad, y ello requiere de trabajadores comprometidos con su organización (Gibson, Ivancebich, y Donnelly, 1994).

Investigadores del comportamiento humano trabajan para conocer los lazos que los compromete a las personas para mantenerse más cerca, identificados o involucrados a su organización (Dessler, 1996). Ciertamente, este fenómeno es un componente intrínseco tanto para el sujeto que se incorpora a trabajar como para la organización que lo recibe. Se trata de un contrato que va más allá de lo formal y legal, al que se le denomina contrato psicológico. Mientras una persona puede estar obligada formalmente a desempeñar una labor, no necesariamente se liga afectivamente a la organización (Arias Galicia, 1972). El contrato psicológico implica la existencia de un conjunto de expectativas recíprocas y están implícitas dentro de la interacción trabajador-organización que tiene que ver con el compromiso de ambas partes y constituye parte trascendente en la efectividad y competitividad de la organización.

Meyer y Allen (7) definen el compromiso organizacional como un "... estado psicológico que caracteriza la relación entre una persona y una organización", donde las expectativas de ambas partes son muy propias, individuales, diferentes y amplias que casi nunca es posible expresar todos los aspectos en una relación formal (incluso legal).

(1) Presidente del Consejo de Transferencia e Innovación, UNMSM

E-mail: alolip@unmsm.edu.pe, alolip@yahoo.es 
Mathieu y Zajac (1990), después de revisar los escritos de un conjunto de investigadores llegaron a señalar que las definiciones sobre compromiso tienen en común expresiones como "... vínculo o lazo del individuo con la organización” (en Bayona, C. y otros, 2000) (4).

Existen otras definiciones últimas sobre compromiso organizacional y todas ellas mantienen el sentido del vínculo y la afectividad como característica básica: “...apego afectivo" (Bayona, C.,Goñi, S. y Madorrán, C., 2000); "...estado interno de la persona" (Lagomarsino, R., 2003), “...vínculos que mantienen a un sujeto apegado a una empresa (Arciniega, L. s/f), "...deber moral o psicológico adquirido hacia una persona, una situación o una institución (Arias Galicia, F. 2003), entre otros (3).

Así, Steers (1977) definió el compromiso como "... la fuerza relativa de identificación y de involucramiento de un individuo con una organización". Tomando como modelo tres fuentes del compromiso: Características personales, características del trabajo y experiencias en el trabajo; se puso en prueba el modelo y se encontró que las organizaciones pueden influir más fácilmente sobre las dos últimas fuentes, en cambio transformar la personalidad sería mucho más difícil y cuestionable desde un ángulo ético.

Entre tanto, Meyer y Allen (7), tras definir el compromiso (señalado antes), indicaron que dicha relación entre una persona y una organización, presenta consecuencias respecto a la decisión para continuar en la organización o dejarla. Estos autores propusieron tres componentes del compromiso: Afectivo (lazos emocionales que las personas forjan con la organización al satisfacer sus necesidades y expectativas), de continuidad o conveniencia (conciencia de la persona respecto a la inversión en tiempo y esfuerzo que se perderían en caso de dejar la organización para encontrar otro empleo) y normativo (la creencia en la lealtad a la organización en correspondencia a ciertas prestaciones).

Entre tanto, existen otros autores que plantean propuestas que difieren en los términos, pero que coinciden en su contenido. Así se encuentra la propuesta de Lagomarsino, R. (2003): compromiso económico, de crecimiento y moral; lo presentado por Bayona, C. y otras (2000): compromiso actitudinal, el calculativo y el normativo, orientado en el mismo sentido de la propuesta de Mathieu y Zajac (1990): compromiso actitudinal y calculador; o el compromiso de identificación, de membresía y de lealtad de Claure Rocha, M. y Böhrt,M. (2003), o compromisos múltiples de Reichers (1985, en Varona, F. 1993). Excepto esta última propuesta, todas son similares a lo planteado por Meyer yAllen (7).
Por su lado, las investigaciones han demostrado que existe relación entre las dimensiones propuestas por Meyer y Allen (1991), probablemente la dimensión que mayor controversia ha suscitado es el compromiso afectivo y continuo (Antón, C. y Gonzáles, L., 2005) (1), cuya relación es moderada. Un estudio sobre el compromiso organizacional como herramienta de gestión de recursos humanos, en una muestra de 138 docentes de la Universidad de Navarra, España, concluye que no existe correlación entre compromiso actitudinal y compromiso de continuidad (nótese que el compromiso actitudinal como el de continuidad se conceptualizan en los términos de Meyer y Allen); que las variables relacionadas con el trabajo y el grupo son las que presentan valores más altos con el compromiso actitudinal, así como la edad y no otra variable resulta significativa en este estudio (Bayona, C., 2000) (4).

Otro trabajo desarrollado sobre compromiso personal hacia la organización y la intención de permanencia, en una muestra de 177 empleados de una institución privada de beneficencia de México, arrojó que el factor más importante para lograr el compromiso e incrementar la intención de permanencia es el apoyo organizacional, siendo éste preponderante sobre el compromiso afectivo, y este último a su vez ejerce mayor peso sobre la satisfacción general en el trabajo de los empleados; finalmente, la expectativa de recompensa quedó en segundo lugar en cuanto a la influencia sobre el compromiso normativo (Arias Galicia, F., 2001) (2).

Claure Rocha, M. y Börht Pelaez, M. (2003), también investigaron sobre las tres dimensiones del compromiso organizacional: identificación, membresía y lealtad, en una muestra de 26 sujetos $(16.25 \%)$ de un total de 160 trabajadores de una empresa privada de servicios de Bolivia, concluyendo que el componente identificación tiene una relación significativa con la variable compromiso organizacional, que el componente membresía tiende a ser débil y poco desarrollado en el grupo estudiado, y que el componente lealtad es más alto en el grupo de 3 a 6 años de servicio en la organización. Así mismo, se encontró correlación significativa entre antigüedad e identificación, entre antigüedad y lealtad $y$, entre antigüedad y compromiso organizacional (5).

En el Perú destaca la investigación desarrollada por Arias, F.; Valega, D.; Loli, A. y Quintana, M. (2003) sobre compromiso organizacional y su relación con algunos factores demográficos, en una muestra de 190 sujetos de diversas organizaciones privadas y públicas y de diferentes tamaños. Los autores encontraron una correlación alta entre compromiso afectivo y satisfacción general con el trabajo, también entre compromiso afectivo y compromiso con el 


\begin{tabular}{|l|c|c|c|c|}
\hline \multicolumn{5}{|c|}{ Cuadro 1. Muestra de estudio } \\
\hline $\begin{array}{l}\text { Variables } \\
\text { demográficas }\end{array}$ & \multicolumn{3}{|c|}{ Grupos de Estudio } & Muestra \\
\hline SEXO & $\begin{array}{c}\text { Femenino } \\
19\end{array}$ & $\begin{array}{c}\text { Masculino } \\
52\end{array}$ & & 71 \\
\hline $\begin{array}{l}\text { ESTADO } \\
\text { CIVIL }\end{array}$ & $\begin{array}{c}\text { Unido } \\
49\end{array}$ & $\begin{array}{c}\text { No unido } \\
22\end{array}$ & & 71 \\
\hline $\begin{array}{l}\text { ESTUDIOS } \\
\text { ALCANZADOS }\end{array}$ & $\begin{array}{c}\text { Universitario } \\
26\end{array}$ & $\begin{array}{c}\text { Técnico } \\
9\end{array}$ & $\begin{array}{c}\text { Otros } \\
36\end{array}$ & 71 \\
\hline
\end{tabular}

trabajo, igual que compromiso afectivo y claridad del rol, en cambio no fue significativa la relación con jerarquía del puesto. Por otro lado, las correlaciones entre el compromiso de conveniencia y las demás variables resultaron inferiores, siendo la más alta con claridad del rol, seguida por satisfacción con el trabajo. En cambio ninguna de las variables demográficas denotó asociación importante con el compromiso de conveniencia (3).

Las organizaciones en el país se están involucrando en una carrera hacia la calidad en los servicios y en la producción de bienes, así como en la gestión. El compromiso de las personas con la organización es una forma de lograr efectividad y calidad en las organizaciones para ser competitivas en el mercado, por lo que, conocer el compromiso de sus colaboradores es vital. Esta investigación pretende ampliar sus hallazgos en una nueva muestra que permita la posibilidad de confirmar estudios anteriores en muestras diferentes.

\section{METODOLOGÍA}

\section{Muestra}

La muestra estuvo conformada por 72 personas de un total de 270 trabajadores no docentes de una Universidad Pública del interior del país, vale decir, el $26.5 \%$, seleccionados al azar. Por razones de limpieza de la muestra se eliminó a un sujeto por no consignar sus datos completos para el análisis de las variables demográficas. Como se puede observar en el cuadro 1, el estado civil consigna los grupos de unidos (que incluye a casados y convivientes) y no unidos (que incluye a divorciados, viudos y solteros) para manejar un mayor número de casos por cada celda. Del mismo modo, se procedió en grado de estudios alcanzado: universitario (incluye a profesionales, a sujetos con estudios concluidos y no concluidos), técnico (incluye a trabajadores que han realizado estudios académicos de una ocupación y a aquellos que se han especializado en la práctica y que se le considera como técnico en planilla) y otros (cualquier trabajador sin estudios universitarios 0 técnicos).

\section{Técnica de recolección de datos}

La técnica utilizada para el acopio de la información fue la administración de la prueba en forma colectiva. La aplicación del instrumento estuvo a cargo de una funcionaria de la Oficina de Recursos Humanos. Se les entregó el cuestionario en un ciclo de conferencias realizado en el auditorio de la institución y recogidos luego. Es posible que esta situación impidiera la revisión minuciosa de cada uno de los cuestionarios para determinar si los datos consignados estaban completos. De manera que, para efectos del análisis se tuvo que eliminar algunos casos.

\section{Instrumento}

El estudio se llevó a cabo con un cuestionario adaptado por Alejandro E. Loli Pineda (2005), con fines de investigación en una realidad distinta a la presente, sobre la base del instrumento preparado por Fernando Arias Galicia, cuyo contenido estaba conformado por reactivos relativos a Compromiso Afectivo, de Conveniencia o continuidad y Sentimiento de Permanencia o normativo (Meyer y Allen, 1991); Satisfacción General en el Trabajo (Eisenberger, 1997), referido a respuestas afectivas dadas en torno a su centro de trabajo; Claridad del Rol (Brown y Leigh, 1996), referido a la diafanidad de las expectativas así como a las normas de trabajo consistentes; Compromiso con el Trabajo (Lodahl y Kejner, 1965), referido al grado en el cual la persona ha acogido los valores respecto a la bondad de sus labores y la importancia de las mismas para su propia valía (6).

\section{Validez factorial}

Aplicado el instrumento y sometido a Análisis Factorial exploratorio (Rotación Varimax con Kaise) permitió confirmar los factores teóricos e identificar otros factores, probablemente debido a las influencias de nuestro contexto socio cultural. Los componentes señalados antes en el cuestionario trabajado por Fernando Arias Galicia (2001) difieren muy sutilmente de los factores extraidos, lo que permite dar fe de su validez confirmatoria.

Así, la variable Claridad del rol apareció difusa y se procedió a no tomar en cuenta dichos ítemes, surgiendo en cambio un nuevo factor al que denominamos Sentimiento de Permanencia (sin duda, equivalente a compromiso normativo), referido a permanecer horas adicionales diarias o semanales y sentirse bien por ello, tal vez por el sentido del deber o en compensación con las prestaciones recibidas, otros dirían por lealtad, en el sentido de cumplimiento y respeto a la organización (Claure, M., 2003). Igualmente se eliminaron algunos itemes de Satisfacción general en el trabajo y otros. En el primer caso, por que el peso factorial era inferior a 0.46 y los predictores de dicho factor eran proporcionalmente 


\begin{tabular}{|l|c|c|c|}
\hline \multicolumn{4}{|c|}{$\begin{array}{c}\text { Cuadro 2. Compromiso organizacional } \\
\text { total de los trabajadores }\end{array}$} \\
\hline & \multicolumn{3}{|c|}{ CATEGORÍAS } \\
\hline & $\begin{array}{r}<\leq \mathrm{Q} 1 \\
\text { (Bajo) }\end{array}$ & $\begin{array}{c}>\text { Q 1 y }<\leq \mathrm{Q} 3 \\
\text { (Normal) }\end{array}$ & $\begin{array}{c}>\mathrm{Q} 3 \\
\text { (Alto) }\end{array}$ \\
\hline Frecuencia & 19 & 35 & 18 \\
\hline Porcentaje & $26 \%$ & $49 \%$ & $25 \%$ \\
\hline
\end{tabular}

excesivos en relación a los demás; en los otros casos, porque no alcanzaron un peso superior a 0.20.

El cuestionario quedó finalmente constituido por 89 reactivos de un total original de 106, quedando conformado por los siguientes factores: Satisfacción general en el trabajo, Compromiso con el trabajo, Compromiso de conveniencia, Compromiso afectivo, Sentimiento de permanencia, Jerarquía del puesto.

\section{Confiabilidad}

De acuerdo al coeficiente alpha de Crombach los diferentes factores cumplen con el criterio de estabilidad y confiabilidad, llegando a un coeficiente de $\leq 0.88$ (A. Loli, 2005), altamente confiable.

\section{RESULTADOS}

\section{Compromiso organizacional de los trabajadores}

El cuadro 2 muestra en términos generales que el mayor porcentaje de personas se ubican dentro de una categoría normal de compromiso organizacional $(49 \%)$, seguido de un bajo compromiso organizacional ( $26 \%$ de sujetos) y un compromiso organizacional alto solo en un $25 \%$ de participantes.

Se puede observar que el puntaje del compromiso organizacional sigue una distribución normal (prueba de Anderson`s T, $p>0.05$ ), siendo el promedio de 404.3 y una mediana de 401.5.

\begin{tabular}{|c|c|c|c|c|c|c|c|}
\hline N Casos & Promedio & Mediana & D.S. & Mínimo & Máximo & Cuartil 1 (Q1) & Cuartil $3($ Q3) \\
\hline 72 & 404.33 & 401.5 & 40.471 & 307 & 528 & 354.8 & 429 \\
\hline
\end{tabular}

\section{Compromiso organizacional general segun variables demográficas}

\section{Compromiso organizacional y sexo}

El Cuadro 3 analiza la relación de los puntajes de compromiso organizacional en hombres y mujeres con un nivel de confianza de $95 \%$ a través de la prueba Chi Cuadrado (razón de verosimilitud) en una muestra total de 71 trabajadores. En esta relación, el $50 \%$ de los

\begin{tabular}{|c|c|c|c|c|}
\hline \multicolumn{5}{|c|}{$\begin{array}{c}\text { Cuadro } 3 \text {. Compromiso organizacional } \\
\text { general según sexo }\end{array}$} \\
\hline \multirow[b]{2}{*}{ SEXO } & \multicolumn{3}{|c|}{ CATEGORIAS } & \multirow[b]{2}{*}{ TOTAL } \\
\hline & $\begin{array}{l}<=Q 1 \\
\text { (Bajo) }\end{array}$ & $\begin{array}{c}>\mathrm{Q} 1 \text { y }<=\mathrm{Q} 3 \\
\text { (Normal) }\end{array}$ & $\begin{array}{l}>\text { Q } 3 \\
\text { (Alto) }\end{array}$ & \\
\hline $\begin{array}{cc}\text { FEMENINO } \mathrm{n}_{\%} \\
\end{array}$ & $\begin{array}{c}3 \\
15.8 \% \\
\end{array}$ & $\begin{array}{c}9 \\
47.4 \% \\
\end{array}$ & $\begin{array}{c}7 \\
36.8 \% \\
\end{array}$ & $\begin{array}{c}19 \\
100 \% \\
\end{array}$ \\
\hline MACULINO $\mathrm{n}_{\%}$ & $\begin{array}{c}15 \\
28.8 \% \\
\end{array}$ & $\begin{array}{c}26 \\
50.0 \% \\
\end{array}$ & $\begin{array}{c}11 \\
21.2 \% \\
\end{array}$ & $\begin{array}{c}52 \\
100 \% \\
\end{array}$ \\
\hline TOTAL & $\begin{array}{c}18 \\
25.4 \% \\
\end{array}$ & $\begin{array}{c}35 \\
49.3 \% \\
\end{array}$ & $\begin{array}{c}18 \\
25.4 \% \\
\end{array}$ & $\begin{array}{c}71 \\
100 \% \\
\end{array}$ \\
\hline
\end{tabular}

varones muestra un compromiso organizacional "normal", siendo éste superior a las mujeres (47.4\%). Sin embargo, de los que tienen compromiso organizacional "alto", la mayoría son mujeres $(36.8 \%)$ en relación a los hombres $(21.2 \%)$. Situación inversa se presenta entre los que tienen compromiso organizacional "bajo", la mayoría son varones $(28.8 \%)$ en relación a las mujeres $(15.8 \%)$.

A pesar de la tendencia expresada, esta relación no es significativa ( $p$-value 0.316 ). Es decir, el sexo no es una variable diferenciadora.

\section{Compromiso organizacional y estado civil}

El cuadro 4 analiza la relación de los puntajes de compromiso organizacional según estado civil: unidos (casados y convivientes) y no unidos (solteros, divorciados y viudos), con un nivel de confianza de $95 \%$ a través de la prueba Chi Cuadrado (razón de verosimilitud) en una muestra total de 71 trabajadores. En esta relación, el $51 \%$ de los unidos muestra un compromiso organizacional "normal", siendo éste superior a las no unidos $(45.5 \%)$. La misma tendencia se observa entre los que tienen compromiso organizacional "alto", la mayoría son los unidos $(26.5 \%)$ en relación a los no unidos $(22.7 \%)$. Situación inversa se presenta entre los que tienen compromiso organizacional "bajo", la mayoría son los no unidos $(31.8 \%)$ en relación a los unidos (22.4\%).

A pesar de la tendencia expresada, esta relación no es significativa ( $p$-value 0.708). Es decir, el estado civil no es una variable diferenciadora.

\begin{tabular}{|c|c|c|c|c|}
\hline \multicolumn{5}{|c|}{$\begin{array}{c}\text { Cuadro 4. Compromiso organizacional } \\
\text { general según estado civil. }\end{array}$} \\
\hline \multirow[b]{2}{*}{ ESTADO CIVIL } & \multicolumn{3}{|c|}{ CATEGORIAS } & \multirow[b]{2}{*}{ TOTAL } \\
\hline & $\begin{array}{l}<=Q 1 \\
\text { (Bajo) }\end{array}$ & $\begin{array}{l}>\mathrm{Q} 1 \mathrm{y}<=\mathrm{Q} 3 \\
\text { (Normal) }\end{array}$ & $\begin{array}{l}>Q 33 \\
\text { (Alto) }\end{array}$ & \\
\hline UNIDOS & $\begin{array}{c}11 \\
22.4 \% \\
\end{array}$ & $\begin{array}{c}25 \\
51.0 \%\end{array}$ & $\begin{array}{c}13 \\
26.5 \%\end{array}$ & $\begin{array}{c}19 \\
100 \%\end{array}$ \\
\hline $\begin{array}{ll}\text { NO UNICOS } & n \\
\%\end{array}$ & $\begin{array}{c}7 \\
31.8 \%\end{array}$ & $\begin{array}{c}10 \\
45.5 \%\end{array}$ & $\begin{array}{c}5 \\
22.7 \%\end{array}$ & $\begin{array}{c}52 \\
100 \%\end{array}$ \\
\hline TOTAL & $\begin{array}{c}18 \\
25.4 \%\end{array}$ & $\begin{array}{c}35 \\
49.3 \%\end{array}$ & $\begin{array}{c}18 \\
25.4 \%\end{array}$ & $\begin{array}{c}71 \\
100 \%\end{array}$ \\
\hline
\end{tabular}


Cuadro 5. Compromiso organizacional general según grado de estudios alcanzado

\begin{tabular}{|c|c|c|c|c|c|}
\hline \multirow{2}{*}{\multicolumn{2}{|c|}{$\begin{array}{l}\text { GRADO DE } \\
\text { ESTUDIOS }\end{array}$}} & \multicolumn{3}{|c|}{ CATEGORIAS } & \multirow[b]{2}{*}{ TOTAL } \\
\hline & & $\begin{array}{l}<=Q 1 \\
\text { (Bajo) }\end{array}$ & $\begin{array}{c}>\mathrm{Q} 1 \mathrm{y}<=\mathrm{Q} 3 \\
\text { (Normal) }\end{array}$ & $\begin{array}{l}>\text { Q } 3 \\
\text { (Alto) }\end{array}$ & \\
\hline Universitario & $\begin{array}{l}\mathrm{n} \\
\% \\
\end{array}$ & $\begin{array}{c}8 \\
30.8 \% \\
\end{array}$ & $\begin{array}{c}11 \\
42.3 \% \\
\end{array}$ & $\begin{array}{c}7 \\
26.9 \% \\
\end{array}$ & $\begin{array}{c}26 \\
100 \% \\
\end{array}$ \\
\hline Técnico & $\begin{array}{l}\mathrm{n} \\
\% \\
\end{array}$ & $22.2 \%$ & $\begin{array}{c}4 \\
44.4 \% \\
\end{array}$ & $33.3 \%$ & $100 \%$ \\
\hline Otros & $\begin{array}{r}n \\
\% \\
\end{array}$ & $\begin{array}{c}8 \\
22.2 \% \\
\end{array}$ & $\begin{array}{c}20 \\
55.6 \% \\
\end{array}$ & $\begin{array}{c}8 \\
22.2 \% \\
\end{array}$ & $\begin{array}{c}36 \\
100 \% \\
\end{array}$ \\
\hline TOTAL & $\mathrm{n}_{\%}$ & $\begin{array}{c}18 \\
25.4 \% \\
\end{array}$ & $\begin{array}{c}35 \\
49.3 \% \\
\end{array}$ & $\begin{array}{c}18 \\
25.4 \% \\
\end{array}$ & $\begin{array}{c}71 \\
100 \% \\
\end{array}$ \\
\hline
\end{tabular}

$\mathbf{p}>0.05$

Tabla de significancia de las variables demográficas respecto al compromiso organizacional

\begin{tabular}{|c|c|c|c|}
\hline FACTORES & $\begin{array}{c}\text { Chi-square } \\
\text { (raz) }\end{array}$ & p-value & Significancia \\
\hline SEXO & 2.302 & 0.316 & n.s. \\
\hline $\begin{array}{c}\text { ESTADO } \\
\text { CIVIL }\end{array}$ & 0.690 & 0.708 & n.s. \\
\hline $\begin{array}{c}\text { GRADO DE } \\
\text { ESTUDIOS }\end{array}$ & 1.429 & 0.839 & n.s. \\
\hline
\end{tabular}

3. Compromiso organizacional y grado de estudios alcanzado.

El cuadro 5 analiza la relación de los puntajes de compromiso organizacional según grados de estudio alcanzados (universitario, técnico y otros) con un nivel de confianza de $95 \%$ a través de la prueba Chi Cuadrado (razón de verosimilitud) en una muestra total de 71 trabajadores.

En esta relación, el $42.3 \%$ de los que alcanzaron estudios universitarios muestran un compromiso organizacional "normal", siendo éste inferior a las que alcanzaron estudios técnicos $(44.4 \%)$ y éste inferior a los que no lograron estudios universitarios ni técnicos (otros: $55.6 \%$ ). Esta tendencia varía entre los que tienen compromiso organizacional "alto", donde la mayoría son técnicos $(33.3 \%)$ en relación a los que alcanzaron estudios universitarios $(26.9 \%)$ y éstos mayor a los que no alcanzaron ni los estudios técnicos ni los estudios universitarios (22.2\%). Situación diferente se presenta entre los que tienen compromiso organizacional "bajo", la mayoría de ellos son los que alcanzaron estudios universitarios $(30.8 \%)$ en relación a los técnicos $(22.2 \%)$ y otros $(22.2 \%)$

A pesar de la tendencia expresada, esta relación no es significativa ( $p$-value 0.839). Es decir, el grado de estudios alcanzado no es una variable diferenciadora.

\begin{tabular}{|l|c|c|c|}
\hline \multicolumn{4}{|c|}{$\begin{array}{c}\text { Cuadro 6. Correlación entre factores de compromiso } \\
\text { organizacional muestra total }\end{array}$} \\
\hline Factores & r-spearman & p-value & significancia \\
\hline ST vs CT & 0.13 & 0.277 & n.s \\
\hline ST vs CC & -0.263 & 0.025 & ${ }^{*}$ \\
\hline ST vs CA & 0.44 & 0 & ${ }^{*}$ \\
\hline ST vs SP & 0.297 & 0.011 & ${ }^{*}$ \\
\hline ST vs JP & 0.247 & 0.036 & ${ }^{*}$ \\
\hline CT vs CC & -0.277 & 0.055 & ${ }^{*}$ \\
\hline CT vs CA & 0.377 & 0.001 & ${ }^{*}$ \\
\hline CT vs SP & 0.277 & 0.018 & ${ }^{*}$ \\
\hline CT vs JP & 0.076 & 0.526 & n.s \\
\hline CC vs CA & -0.056 & 0.638 & n.s \\
\hline CC vs SP & 0.062 & 0.602 & n.s \\
\hline CC vs JP & 0.111 & 0.353 & n.s \\
\hline CA vs SP & 0.418 & 0 & ${ }^{*}$ \\
\hline CA vs JP & 0.298 & 0.011 & ${ }^{*}$ \\
\hline SP vs JP & 0.272 & 0.021 & $*$ \\
\hline
\end{tabular}

Relación entre los factores de compromiso organizacional de los trabajadores

En el cuadro 6 se observa la relación entre los factores del compromiso organizacional. Para averiguar si existe asociación entre ellos, se utilizó la correlación de Spearman. con un nivel de confianza del $95 \%$, mediante la prueba de Spearman en una muestra total de 71 trabajadores. En esta relación se encontró que la satisfacción general con el trabajo correlaciona positivamente con compromiso afectivo ( $p$-value 0.000$)$, con sentimiento de permanencia ( $p$ value 0.011) y con jerarquía del puesto ( $p$-value 0.036), y esta asociación es significativa.

Lo que quiere decir, que a mayor satisfacción con el trabajo hay mayor compromiso afectivo, mayor sentimiento de permanencia y mayor compromiso con la jerarquía del puesto.

En el mismo cuadro se encontró que el compromiso con el trabajo correlaciona con compromiso afectivo ( $p$-value 0.001) y sentimiento de permanencia ( $p$ value 0.018), y esta asociación es positiva y significativa. Lo que debe entenderse que a mayor compromiso con el trabajo mayor compromiso afectivo y mayor sentimiento de permanencia. Lo mismo ocurre con el compromiso afectivo que correlaciona con sentimiento de permanencia $(p-$ value 0.000$)$ y con jerarquía del puesto ( $p$-value 0.011 ); a su vez, el sentimiento de permanencia correlaciona con jerarquía del puesto ( $p$-value 0.021). 
Cuadro 7. Correlación entre factores del compromiso organizacional

\begin{tabular}{|c|c|c|c|c|c|c|c|c|}
\hline \multicolumn{9}{|c|}{ Correlations } \\
\hline & & & ST & $\mathrm{CT}$ & $\mathrm{CC}$ & CA & SP & JP \\
\hline \multirow[t]{18}{*}{ Spearman's rho } & ST & Correlation Coefficient & 1.000 & .130 & $-.263 *$ & $.440 * *$ & $.297 *$ & $.247 *$ \\
\hline & & Sig. (2-tailed) & & .277 & .025 & .000 & .011 & .036 \\
\hline & & $\mathrm{N}$ & & 72 & 72 & 72 & 72 & 72 \\
\hline & $\mathrm{CT}$ & Correlation Coefficient & & 1.000 & -.227 & $.377 * *$ & $.277 *$ & .076 \\
\hline & & Sig. (2-tailed) & & & .055 & .001 & .018 & .526 \\
\hline & & $\mathrm{N}$ & & & 72 & 72 & 72 & 72 \\
\hline & $\mathrm{CC}$ & Correlation Coefficient & & & 1.000 & -.056 & .062 & .111 \\
\hline & & Sig. (2-tailed) & & & & .638 & .602 & .353 \\
\hline & & $\mathrm{N}$ & & & & 72 & 72 & 72 \\
\hline & $\mathrm{CA}$ & Correlation Coefficient & & & & 1.000 & $.418 * *$ & $.298 *$ \\
\hline & & Sig. (2-tailed) & & & & & .000 & .011 \\
\hline & & $\mathrm{N}$ & & & & & 72 & 72 \\
\hline & SP & Correlation Coefficient & & & & & 1.000 & $.272 *$ \\
\hline & & Sig. (2-tailed) & & & & & & .021 \\
\hline & & $\mathrm{N}$ & & & & & & 72 \\
\hline & JP & Correlation Coefficient & & & & & & 1.000 \\
\hline & & Sig. (2-tailed) & & & & & & \\
\hline & & $\mathrm{N}$ & & & & & & \\
\hline
\end{tabular}

Estas últimas asociaciones son positivas y significativas, por lo que se puede decir que, a mayor compromiso afectivo mayor sentimiento de permanencia y mayor compromiso con la jerarquía del puesto, así como a mayor sentimiento de permanencia mayor compromiso con la jerarquía del puesto.

Por otro lado, también se encontró correlación entre la satisfacción general con el trabajo con compromiso de conveniencia ( $p$-value 0.025 ) y el compromiso con el trabajo con compromiso de conveniencia ( $p$-value $0.0)$ y estas asociaciones son significativas pero negativas; lo que quiere decir, que a mayor satisfacción general con el trabajo menor compromiso de conveniencia, y a mayor compromiso con el trabajo menor compromiso de conveniencia.

Entre los demás factores del compromiso organizacional que no han resultado significativas se encuentran: satisfacción general con el trabajo con compromiso con el trabajo (p-value 0.277), compromiso con el trabajo con jerarquía del puesto (p-value 0.526), compromiso de conveniencia con compromiso afectivo ( $p$-value 0.638), compromiso de conveniencia con sentimiento de permanencia $(p-$ value 0.692), y compromiso de conveniencia con jerarquía del puesto (p-value 0.353).

\section{DISCUSIÓN}

Los resultados muestran que un importante porcentaje de trabajadores tienen una actitud favorable hacia el compromiso organizacional, lo que hace suponer que hay la posibilidad de un comportamiento comprometido con su organización, de acuerdo con la teoría de Azjen y Fishbein (1977, 1980, en Arias, 2003) que señalan que la intención es la antesala del comportamiento. Sin embargo, hay un porcentaje de trabajadores que merecen atención por cuanto su compromiso con la organización es bajo. 
Respecto a la relación del compromiso organizacional con algunas variables demográficas (sexo, estado civil y grado de estudios alcanzado) se puede decir que no arrojan significancia estadística, hallazgo que es coherente con lo encontrado por Arias Galicia, Valera Sosa y Loli Pineda (2003), en una muestra variada y por A. Loli Pineda (2005) en una muestra de trabajadores de 20 Facultades de una universidad pública de Lima. Lo que indica que algunas variables demográficas no constituyen elementos diferenciadores cuando se trata del compromiso organizacional; en otros términos, el compromiso organizacional es igual para los trabajadores.

Revisando la correlación entre los factores del compromiso organizacional se ve una asociación altamente significativa entre satisfacción general con el trabajo con compromiso afectivo y éste con sentimiento de permanencia, igualmente, compromiso con el trabajo con compromiso afectivo. Entre tanto, existe asociación menor pero significativa entre satisfacción general con el trabajo con sentimiento de permanencia y jerarquía del puesto, compromiso afectivo con sentimiento de permanencia y jerarquía del puesto y entre sentimiento de permanencia y jerarquía del puesto, hallazgo que se ratifica con lo encontrado por Arias Galicia (2001), excepto la asociación con el compromiso con la jerarquía del puesto que se añade en este caso. Igualmente, es significativa la asociación entre satisfacción general con el trabajo con compromiso de conveniencia y entre éste con compromiso con el trabajo, siendo estas dos últimas asociaciones negativas.

Estos últimos hallazgos confirman lo encontrado por A. Loli Pineda (2005) en una muestra similar pero en una universidad pública de Lima y puede estar relacionado como dice el autor a las condiciones económicas y las condiciones laborales que se les ofrece y el alto índice de desempleo del mercado laboral, comportamiento coherente con la revisión de las investigaciones practicadas y su propuesta del compromiso calculador de Mathieu y Zajac (1990), los hallazgos de Bayona, C., y otros (2000) y la teoría de Meyer y Allen (1991) en el sentido de condicionar su desempeño al costo de su inversión (Becker, 1960) o a la presión del desempleo del mercado (Vandenberghe, 1996).

Como se puede apreciar, los resultados son indefectiblemente consistentes a pesar de las limitaciones descritas sobre las condiciones en que fue administrada la prueba a los participantes de la muestra de estudio. Sin duda, sería pertinente buscar asociación del compromiso organizacional con otras variables como valores organizacionales, la calidad del servicio o con aquellas más personales como la autoestima, por que el compromiso organizacional involucra variables que van más allá de la edad, el sexo y otras variables demográficas, que no han mostrado aún relevancia importante de asociación hasta ahora.

\section{CONCLUSIONES}

En términos generales, el mayor porcentaje de personas se ubican dentro de una categoría normal de compromiso organizacional, seguido de otro grupo con un bajo compromiso organizacional y un tercer grupo menor que el anterior con un compromiso organizacional alto.

Por otro lado, no existe relación entre el compromiso organizacional y las variables demográficas: sexo, estado civil y grado de estudios alcanzado. Es decir, estas variables demográficas no son variables diferenciadoras. No existe cambio alguno con la intervención de estas variables.

Asimismo, un análisis de los factores de compromiso organizacional conduce a afirmar que existe asociación con algunas variables organizacionales:

a) Existe asociación entre la satisfacción general con el trabajo con compromiso afectivo, con sentimiento de permanencia y jerarquía del puesto.

b) Existe asociación entre compromiso con el trabajo con compromiso afectivo y sentimiento de permanencia.

c) Existe asociación entre compromiso afectivo con sentimiento de permanencia con jerarquía del puesto, así como el sentimiento de permanencia con jerarquía del puesto.

d) Existe asociación entre satisfacción general con el trabajo con compromiso de conveniencia, y entre compromiso con el trabajo con compromiso de conveniencia pero negativa.

\section{REFERENCIAS BIBLIOGRÁFICAS}

1. Antón, C. y Gonzáles, L. (2005). Examen de las relaciones entre el compromiso organizacional afectivo y continuo. España, Universidad de Salamanca.

2. Arias Galicia, F. (2001). El compromiso personal hacia la organización y la intención de permanencia: algunos factores para su incremento. México, Edit. UNAM. Rev. de Contaduría y Administración, $N^{\circ} 200$, enero marzo 2001.

3. Arias Galicia, F.; Varela Sosa, D.; Loli Pineda, A.; y Quintana Otiniano, M. (2003). El compromiso organizacional y su relación con algunos factores 
demográficos. Lima-Perú: Rev. de Investigación en Psicología, vol. 6, N2 2, UNMSM.

4. Bayona, C., Goñi, S. y Madorrán, C., (2000). Compromiso organizacional: implicaciones para la gestión estratégica de los recursos humanos. www.upna.es.

5. Claure Rocha, M. y Böhrt Pelaez, M. (2003). Tres dimensiones del compromiso organizacional: identificación, membresía y lealtad. Bolivia. Rev. AJAYU, año 2003, Vol. 2, № 1.
6. Loli Pineda, Alejandro (2005). Compromiso organizacional de los trabajadores de una universidad pública de Lima y su relación con algunas variables demográficas. Instituto de Investigaciones Psicológicas, UNMSM, Lima, Perú.

7. Meyer, J.P. y Allen, N.J. (1991). Tres componentes, Conceptualización del compromiso organizacional. Human Resource Management Review, 1, 61-98. 